\title{
Editorial
}

\section{New A\&A editorial policy for papers about instrumentation}

\author{
Claude Bertout ${ }^{1}$ and Malcolm Walmsley ${ }^{2}$ \\ 1 Editor-in-Chief, Astronomy \& Astrophysics \\ 2 Letters Editor-in-Chief, Astronomy \& Astrophysics
}

\begin{abstract}
Astronomy $\mathcal{E}$ Astrophysics introduces a new editorial policy for papers submitted to Section 13 (Astronomical Instrumentation).

Key words. editorials
\end{abstract}

In their research, astronomers rely heavily on new, cutting-edge instruments that make their exciting discoveries possible. The design and construction of instrumentation for ground-based and space telescopes is thus an essential astronomical activity, which includes, besides the instrumental developments themselves, (i) the testing of promising new sites for telescope deployment, (ii) the specific observational procedures and scientific preparatory studies needed to operate the instrumentation in the most efficient way, (iii) the calibration that translates the measurements into physical units, thus permitting a comparison of results obtained by different instruments, $(i v)$ the data processing procedures tailored to the new instruments, and $(v)$ the tools allowing for optimal inter-operability of archived data.

Recognizing the importance of state-of-the-art instrumentation, the A\&A Board of Directors has decided to develop the corresponding journal section, thus aiming at making A\&A a reference journal also for astronomers whose main interest is instrumentation. We therefore introduce hereby the new editorial policy concerning these papers. In Section 13, we will now publish papers that describe

- new concepts and ideas that might lead to actual future instruments,

- crucial instrumental developments in ongoing ground-based or space projects,

- studies that are essential to the preparation of large instrumental projects,

- ground-breaking data processing and mining methods,

provided these works report a significant advance on current capabilities and are of interest to a sizable fraction of the community.

Compared to our previous editorial policy for Section 13, the main change is that we no longer request that papers describing instruments and related studies also present astronomical results.

The peer-review policy for Section 13 papers will remain the same as previously. Referees with strong instrumental background will be requested to scrutinize the submitted manuscripts to ensure the highest possible level of scientific merit for those works that will be accepted for publication. Authors will be expected to write their papers with the large audience of A\&A in mind rather than for a small group of specialists alone.

In order to implement the new editorial policy in a consistent manner, a single Associate Editor will have responsibility for all papers submitted for Section 13.

Following a trend started with A\&A Section 14 (Online catalogs and data), Section 13 will be published mainly online. The first page of Section 13 papers, comprising the title, author names and affiliations, and abstract, will be printed. The electronic edition of the journal, which is now the reference version of A\&A since the printed edition is no longer complete, will contain the full papers and nothing will distinguish online papers from printed ones in this electronic edition. All A\&A papers are, of course, referenced in the Smithsonian/NASA Astrophysics Data System (ADS) and other preprint servers.

Our publisher EDP Sciences has kindly agreed to provide open access to Section 13 articles to promote the instrumental section. In 2007, instrumental articles published in A\&A will thus be accessible to everyone at no cost. 